\title{
Instanton String and M-Wave in Multiple M5-Branes System
}

\author{
Chong-Sun $\mathrm{Chu}^{1,2,3}$ and Hiroshi Isono ${ }^{1}$ \\ ${ }^{1}$ Department of Physics, National Tsing-Hua University, \\ Hsinchu 30013, Taiwan \\ 2 National Center for Theoretical Sciences, National Tsing-Hua University, \\ Hsinchu 30013, Taiwan \\ 3 Centre for Particle Theory and Department of Mathematics, \\ Durham University, Durham, DH1 3LE, UK \\ E-mail: chong-sun.chu@durham.ac.uk, hisono@phys.nthu.edu.tw
}

\begin{abstract}
We consider the non-abelian self-dual two-form theory $\square 1$ and find new exact solutions. Our solutions are supported by Yang-Mills (anti)instantons in 4-dimensions and describe wave moving in null directions. We argue and provide evidence that these instanton string solutions correspond to M-wave (MW) on the worldvolume of multiple M5-branes. When dimensionally reduced on a circle, the MW/M5 system is reduced to the D0/D4 system with the D0-branes represented by the Yang-Mills instanton of the D4-branes Yang-Mills gauge theory. We show that this picture is precisely reproduced by the dimensional reduction of our instanton string solutions.
\end{abstract}

Keywords: M-Theory, D-branes, M-branes, Gauge Symmetry. 


\section{Introduction}

The theory of $N$ coincident M5-branes in a flat spacetime is given by an interacting $(2,0)$ superconformal theory in six dimensions [2]. The understanding of the dynamics of this system is of utmost importance. On general grounds, the theory does not have a free dimensionless parameter and is inherently non-perturbative. It does not mean that an action does not exist, though it does mean that the use of the action will be limited to non-perturbative analysis, for example, the studies of solutions to the equations of motion. This is still very interesting, particularly since much of the spacetime M-theory physics can be learnt from the physics of the solitonic objects of the the worldvolume theory of M5-branes. This is of course valid also for M2-branes as well as D-branes in string theory and this kind of target space-worldvolume duality has dominated the developments of string theory in the last 15 years or so, with the AdS/CFT correspondence [3] being the most celebrated duality.

For a single M5-brane, the equation of motion has been constructed [4-7]. For multiple M5-branes, a major difficulty has been to non-abelianize the self-dual tensor gauge dynamics. This problem was tackled and a solution was presented in [1] where a consistent self-duality equation of motion for the non-abelian tensor gauge field was constructed. Moreover it was proposed as the low energy equation of motion of the self-dual tensor field living on the worldvolume of a system of multiple M5-branes.

The construction of [1] consists of two primary steps: the identification of the form of the non-abelian gauge symmetry and the construction of the dynamical self-duality equation. The proposed form of the non-abelian gauge symmetry was motivated by the analysis in [8] where a set of 5-dimensional non-abelian one-form gauge fields was introduced in order to incorporate non-trivial interactions among the 2-form potentials [9]. The introduction of the non-abelian 1-form gauge fields allows one to write covariant derivative and to introduce non-abelian transformation for the fields ${ }^{1}$. However since there is no room for propagating 1 -form gauge fields in the $(2,0)$ self-dual tensor multiplet in 6-dimensions, they must be constrained and be non-propagating ${ }^{2}$.

The constraint needed for the specification of the gauge symmetry was identified in [1]. There, a self-duality equation for a non-abelian 2-form in 6-dimensions was

\footnotetext{
${ }^{1}$ Similar forms of gauge symmetry were also considered by [10], as well as [11] where an extra 3 -form potential was introduced in addition to the propagating 1-form gauge potential. The latter formulation were further developed in $[12,13]$ and provides a construction for a class of $(1,0)$ superconformal models in 6-dimensions.

${ }^{2}$ The philosophy is very similar to the BLG [14] and ABJM model [15] of multiple M2-branes where a set of non-propagating Chern-Simons gauge fields was introduced in order to allow for a simple representation of the highly non-linear and non-local self interactions of the matter fields of the $\mathcal{N}=8$ supermultiplet in 3 -dimensions.
} 
constructed in the gauge $B_{5 \mu}=0(\mu=0, \cdots, 4)$ and reads

$$
\tilde{H}_{\mu \nu}=\partial_{5} B_{\mu \nu}
$$

The constraint for the gauge field $A_{\mu}$ is given by

$$
F_{\mu \nu}=c \int d x_{5} \tilde{H}_{\mu \nu}
$$

Here

$$
\begin{gathered}
H_{\mu \nu \rho}=D_{[\mu} B_{\nu \rho]}=\partial_{[\mu} B_{\nu \rho]}+\left[A_{[\mu}, B_{\nu \rho]}\right], \\
\tilde{H}_{\mu \nu}=-\frac{1}{6} \epsilon_{\mu \nu \rho \sigma \tau} H^{\rho \sigma \tau}, \quad \epsilon_{01234}=-1, \\
F_{\mu \nu}=\partial_{\mu} A_{\nu}-\partial_{\nu} A_{\mu}+\left[A_{\mu}, A_{\nu}\right]
\end{gathered}
$$

and $c$ is a constant that is fixed by quantization condition of the self-dual strings solution of the theory $[16,17]$. All fields are in the adjoint representation of the Lie algebra of the gauge group $G$. The self-duality equation (1.1) and the constraint (1.2) were derived in [1] as the equations of motion of an action principle, which is a generalization of the abelian theory of Perry-Schwarz [5]. Evidence that this self-duality equation describes physics of M5-branes was provided in [1], and further in $[16,17]$ where non-abelian self-dual string solutions were constructed. In these latter two papers, it was argued that the form of the 1/2 BPS equation with a single scalar activated could be derived from the requirement of conformal symmetry and R-symmetry of the system and it was shown that the solution of the pure gauge sector could be lifted to become a solution of the non-abelian $(2,0)$ theory with selfdual electric and magnetic charges. In M-theory, the self-dual string arises from the intersection of a system of M2-branes with the system of multiple M5-branes. It is satisfying that the constant $c$ is fixed, which would otherwise be a free dimensionless constant in the theory and hence contradicts with what we know about M5-branes in flat space. It is also quite encouraging that a complete agreement [17] of the field theory results and the supergravity descriptions [18] was found, thereby providing substantial support to the proposal of [1] that (1.1) is the self-duality equation for multiple M5-brane system.

We remark that in these solutions, the self-dual strings were uniform and static and lie in the $x^{0}$ and $x^{4}$ directions. As a result, the solutions take the form with $B=B\left(x^{i}, x^{5}\right), A=A\left(x^{i}\right), i=1,2,3$. It is interesting that the self-dual string solutions found in $[16,17]$ were supported by having its auxiliary gauge field $A$ given by a non-abelian monopole configuration in 3-dimensions $x^{i}$, and that the charge of the self-dual strings is given by the monopole charge. Motivated by this observation, 
it is natural to ask if there are other exact solutions of the non-abelian self-duality equation (1.1) that are supported by other interesting gauge field configurations; and if so, what are their interpretations in M-theory?

In the next section, we construct a new class of solutions of the self-duality equation obtained by having its auxiliary gauge fields given by Yang-Mills (anti)instantons in 4-dimensions. We will argue that the solutions describe M-wave on M5-branes. The paper is concluded with some further discussions in section 3.

\section{M-Wave Solution}

Throughout the paper we follow the convention of $[1,16,17]$. In particular the $5 \mathrm{~d}$ and $6 \mathrm{~d}$ coordinates are denoted by $x^{\mu}=\left(x^{0}, x^{1}, x^{2}, x^{3}, x^{4}\right)$ and $x^{M}=\left(x^{\mu}, x^{5}\right)$. We are interested in finding new solutions of the self-duality equation and their M-theory interpretation. Let us first consider the case of non-compact $x^{5}$. The equations read

$$
\begin{gathered}
\tilde{H}_{\mu \nu}=\partial_{5} B_{\mu \nu} \\
F_{\mu \nu}=c\left[B_{\mu \nu}\left(x_{5}=\infty\right)-B_{\mu \nu}\left(x_{5}=-\infty\right)\right] .
\end{gathered}
$$

Splitting $x^{\mu}$ into $x^{0}$ and $x^{a}=\left(x^{1}, x^{2}, x^{3}, x^{4}\right)$, then (2.1) reads

$$
\begin{aligned}
& \tilde{H}_{0 a}=\partial_{5} B_{0 a}=-\frac{1}{6} \epsilon_{a b c d} H_{b c d}, \\
& \tilde{H}_{a b}=\partial_{5} B_{a b}=\frac{1}{2} \epsilon_{a b c d} H_{0 c d} .
\end{aligned}
$$

Let us take an ansatz $B_{0 a}=0, A_{0}=0$. We have

$$
\begin{aligned}
0 & =-\frac{1}{6} \epsilon_{a b c d} H_{b c d}, \\
\partial_{5} B_{a b} & =\frac{1}{2} \epsilon_{a b c d} \partial_{0} B_{c d} .
\end{aligned}
$$

The equation (2.5) can be solved with

$$
B_{a b}=F_{a b} f\left(x^{0}, x^{5}\right)
$$

for arbitrary $A_{a}$ due to the Bianchi identity of $F_{a b}$. If we further assume that $A_{a}$ 's are independent of $x^{0}$, then (2.6) reads

$$
F_{a b} \partial_{5} f=\frac{1}{2} \epsilon_{a b c d} F_{c d} \partial_{0} f .
$$


This can be solved with

$$
\begin{aligned}
F_{a b} \text { being SD and } f & =f\left(x^{0}+x^{5}\right), \\
\text { or, } F_{a b} \text { being ASD and } f & =f\left(x^{0}-x^{5}\right),
\end{aligned}
$$

where (A)SD stands for (anti)self-dual. Finally the equation (2.2) requires that

$$
c[f(\infty)-f(-\infty)]= \pm 1
$$

where + is for the SD case and - is for the ASD case. For example, one can take $f=\tanh \left(x^{0} \pm x^{5}\right) /(2 c)$. Since our solution is supported by an instanton configuration, it might be called an instanton string. What kind of M-theory object does it describe?

In M-theory, there are four basic objects preserving half of the supersymmetries. These are the M5-brane, M2-brane, M-wave (MW) and the Kaluza-Klein monopole $(\mathrm{MK})$. The M-wave solution in supergravity was originally constructed in [19]. For objects with an extended longitudinal space, it is possible to turn on a wave in a null direction along the brane. For example, one can have an MW in the $x^{0}, x^{5}$ directions on a system of M5-branes. The intersecting brane system is $1 / 4$ BPS in the 11-dimensional supergravity and a smeared (delocalized) supergravity solution has been constructed in [20]. We propose that our instanton string solution is describing a MW on a system of multiple M5-branes.

To check this, let us consider supersymmetry. We remark that the abelian $(2,0)$ tensor multiplet has the supersymmetry transformation

$$
\begin{aligned}
\delta X^{I} & =i \bar{\epsilon} \Gamma^{I} \Psi \\
\delta \Psi & =\Gamma^{M} \Gamma_{I} \partial_{M} X^{I} \epsilon+\frac{1}{3 !} \frac{1}{2} \Gamma^{M N L} H_{M N L} \epsilon, \\
\delta B_{M N} & =i \bar{\epsilon} \Gamma_{M N} \Psi,
\end{aligned}
$$

where $I, J=6,7, \cdots, 10$ and $M, N, L=0,1, \cdots, 5$. For the non-abelian case, let us assume that the supersymmetry transformation takes on a similar form plus terms that vanish on our solution. Specifically, let us assume that for $\Psi$,

$$
\delta \Psi=\Gamma^{M} \Gamma_{I} D_{M} X^{I} \epsilon+\frac{1}{3 !} \frac{1}{2} \Gamma^{M N L} H_{M N L} \epsilon+\cdots
$$

where $\cdots$ denotes terms that vanish for our solution, e.g. terms involving two or more different $X$ 's. This form of the supersymmetry has been tested in $[16,17]$. The form (2.14) of supersymmetry is also reasonable since, as a result of our ansatz (2.7), it implies $\delta F_{a b}=\delta B_{a b}=0$ for our solution and this is consistent with the fact that the auxiliary gauge field $A_{a}$ is supposed to be supersymmetry invariant. 
Now, for our solution $X^{I}=0, \Psi=0$ hold, therefore 2.15) implies

$$
0=\left(\Gamma^{0 a b} H_{0 a b}+\Gamma^{5 a b} H_{5 a b}\right) \epsilon
$$

where $H_{5 a b}:=\partial_{5} B_{a b}$. Since our solution satisfies $H_{0 a b}= \pm H_{5 a b}$ with the plus sign for SD $F_{a b}$ and the minus sign for ASD $F_{a b}$, therefore (2.16) implies

$$
\Gamma_{05} \epsilon= \pm \epsilon
$$

with the plus sign for the SD case and the minus sign for the ASD case. The projector condition (2.17) means a breaking of half of the supersymmetry of the $(2,0)$ theory and is in fact precisely the same supersymmetry preserving condition for MW on M5-branes. This supports the identification of our instanton string with the MW.

To further justify our claim, let us consider a compactification of our system on a circle, say, for $x^{5}$ to be compactified on a circle of radius $R$. In the brane picture, the $\mathrm{MW} / \mathrm{M} 5$ system is reduced to a D0/D4 system where the D0-branes are represented as Yang-Mills instanton of the Yang-Mills theory of the D4-branes [21,22] due to the coupling on the D4-brane worldvolume to the RR-gauge field $C_{1}$,

$$
S_{R R} \sim \int d^{5} x C_{1} \wedge \operatorname{tr}(F \wedge F)
$$

The number of D0-branes is given by the instanton number. We will now show that this picture is reproduced precisely by the compactification of our instanton string solution.

For $x^{5}$ being compactified on a circle of radius $R$, the condition (2.2) reads

$$
F_{\mu \nu}=2 \pi R c H_{5 \mu \nu}^{(0)}
$$

where $H_{5 \mu \nu}^{(0)}$ is the zero mode part of the modes expansion of $H_{5 \mu \nu}$. For our ansatz with nonzero $A_{a}$ and $B_{a b}$, the equations (2.5), (2.6) read

$$
\begin{gathered}
\epsilon_{a b c d}\left(D_{b} B_{c d}\right)^{(n)}=0, \\
\left(\partial_{5} B_{a b}\right)^{(n)}=\frac{1}{2} \epsilon_{a b c d}\left(\partial_{0} B_{c d}\right)^{(n)},
\end{gathered}
$$

where $X^{(n)}$ denotes coefficient of the $n$-th Fourier mode of $X=\sum_{n=-\infty}^{\infty} X^{(n)} e^{i n x^{5} / R}$. Consider the ansatz

$$
B_{a b}=\alpha \times\left(x^{5} \pm x^{0}\right) F_{a b}\left(x^{a}\right)+\sum_{n=-\infty}^{\infty} B_{a b}^{(n)}\left(x^{0}, x^{a}\right) e^{i n x^{5} / R}
$$

where $\alpha$ is a constant, $F_{a b}$ is SD for the plus sign and ASD for the minus sign. Note that a linear term in $x^{0}$ and $x^{5}$ is allowed as it is only required that the field strength 
to be a periodic function of $x^{5}$. The form of the linear part is fixed by solving the zeroth part of the equations (2.20), (2.21). As for the Fourier modes, the zeroth mode is required by 2.20 ) to obey $\epsilon_{a b c d} D_{b} B_{c d}^{(0)}=0$ and this can be solved by

$$
B_{a b}^{(0)}=\beta_{0} F_{a b}
$$

where $\beta_{0}$ is a constant. As for the non-zero mode equations, they can be solved with

$$
B_{a b}^{(n)}=\beta_{n} e^{ \pm i n x^{0} / R} F_{a b}
$$

where $F_{a b}$ is SD for the plus sign and ASD for the minus sign and $\beta_{n}$ are constants.

All in all, the dimensionally reduced system (2.20), (2.21) can be solved by

$$
B_{a b}=F_{a b}\left(x^{k}\right) f\left(x^{0} \pm x^{5}\right)
$$

with

$$
f\left(x^{0} \pm x^{5}\right):=\alpha\left(x^{5} \pm x^{0}\right)+\sum_{n=-\infty}^{\infty} \beta_{n} e^{\frac{i n\left(x^{5} \pm x^{0}\right)}{R}},
$$

where $F_{a b}$ is SD for the plus sign and ASD for the minus sign. This is of course nothing but simply the solution (2.7), (2.9), (2.10) with now a profile function $f$ that is consistent with the compactification condition. The constant $\alpha$ can be fixed by noticing that our solution (2.26) gives

$$
H_{5 a b}^{(0)}=\alpha F_{a b}
$$

and the condition (2.19) implies that

$$
\alpha=\frac{1}{2 \pi R c} .
$$

Note that $H_{5 a b}^{(0)}$ is independent of $B^{(0)}$. We also record

$$
H_{5 a b}=F_{a b}\left(\alpha+\sum_{n=-\infty}^{\infty} \frac{i n \beta_{n}}{R} e^{\frac{i n\left(x^{5} \pm x^{0}\right)}{R}}\right) .
$$

Now the general analysis of the dimensional reduction of the non-abelian selfduality equation has been performed in [1]. Note that on dimensional reduction of our non-abelian M5 brane theory, the field strength

$$
F_{\mu \nu}=2 \pi R c H_{5 \mu \nu}^{(0)}
$$


becomes the field strength of the 5d supersymmetric Yang-Mills theory. Indeed it is no longer interpreted as an auxiliary field but becomes propagating and obeys a dynamical equation of motion [1],

$$
D_{\mu} F^{\mu \nu}=-\frac{\pi R}{2} \epsilon^{\nu \alpha \beta \gamma \delta}\left[F_{\alpha \beta}, B_{\gamma \delta}\right]
$$

In general, this is more complicated than the source free Yang-Mills equation. In [1], the additional term on the right hand side was argued to be arising from higher derivatives corrections of the Yang-Mills action. For our specific solution (2.26), the right hand side of (2.31) is zero since $B_{a b} \propto F_{a b}$ in our solution. Therefore, on dimensional reduction, our instanton string gives rise exactly to an instanton (or anti-instanton) configuration in a standard 5-dimensional Yang-Mills action. This is precisely what is expected for the system of D0-branes in D4-branes. Besides, we can see that the RR coupling (2.18) does arise naturally from the non-abelian M5-branes theory on dimensional reduction. In fact, (2.18) is just a part of the coupling of the metric to the energy-momentum tensor

$$
\int d^{6} x g_{05} T^{05}
$$

since $C_{1} \sim g_{05}$ and quite generally one can expect the energy-momentum tensor of the system of multiple M5-branes to contain the term (up to a normalization constant),

$$
T^{M N}=\operatorname{tr}\left(H^{M P Q} H_{P Q}^{N}\right)+\cdots
$$

This is the case for the abelian M5-brane [23] and for the non-abelian (2,0) theory [24]. And it should be true irrespective of whether the theory is self-dual or not.

\section{Discussions}

In this paper, we have constructed a new solution of the non-abelian self-duality equation. The solution is supported by an instanton or anti-instanton configuration for the auxiliary Yang-Mills gauge field. We have argued and provided evidence that our solution provides a field theory description of the M5/MW intersecting system. Together with other evidence provided previously $[1,16,17]$, we are quite confident of (1.1), (1.2) as providing a description of the self-dual dynamics of multiple M5branes.

As instanton, our solution is localized in the Euclidean $x^{a}$ directions. It will be interesting to construct localized supergravity solution for the M5/MW system. It may be possible to build it similarly to the localized supergravity solution of 
intersecting M5-branes [25]. This will provide further features of the solution which one can compare with the field theory results, thus furnishing a more detailed and more interesting test.

It is important to complete the bosonic self-duality equations with supersymmetry. Some difficulties associated with the supersymmetric completion have been discussed in [1]. In our present construction of the instanton string solution, we have provided another valuable piece of information concerning the form of the supersymmetry transformation. It will be important to push this direction forward.

If a $C$-field is turned on in the worldvolume of the system of M5-branes, then one can expect that some kinds of star-product will arise as a result of the emerged noncommutative geometry, and the self-duality equation will be modified. In [26], the logic was reversely applied and it was shown how one can deduce the known form of worldvolume noncommutative geometry of D-branes [27-29] from a knowledge of how the $B$-field modifies the Nahm equation; as well as the noncommutative geometry of M5-brane in a large $C$-field from a knowledge of how the $C$-field modifies the Basu-Harvey equation [30]. The found noncommutative geometry takes the form of a quantum Nambu geometry [31]. However there has not been success in reformulating this operatorial geometry in terms of more familiar language of a deformed starproduct. Knowing how the $C$-field modifies the nonabelian self-duality equation may provide hints to this problem.

In addition to the 5d-SYM proposal $[32,33]$, there exist a number of other earlier proposals $[34,35]$ and [36] for the definition of the theory of multiple M5-branes. These proposals have the advantage of being supposed to be providing a fundamental quantum description, but is however much less explicit. It will be interesting to explore the connection of our description with these proposals.

In some sense, the self-duality equation (1.1) is a higher dimensional generalization of the self-dual Yang-Mills equation in 4-dimensions. The self-dual Yang-Mills equation is an interesting mathematical physics system. Apart from numerous important applications in mathematics, it is exactly solvable. Moreover, it has been conjectured by Ward [37] to be a kind of master equation, which states that all the integrable equations in three of lower dimensions can be obtained from the self-dual Yang-Mills equation by a reduction. It is interesting to ask if the self-duality equation (1.1) has also similarly rich and interesting mathematical properties. For example, is it integrable? is there a twistor construction behind, and is there any connection with the twistor or loop formulation proposed in [38]? We leave these interesting questions for further exploration. 


\section{Acknowledgements}

It is our pleasure to thank Kazuyuki Furuuchi, Sheng-Lan Ko, Christian Saemann, Richard Szabo, Pichet Vanichchapongjaroen and Martin Wolf for discussions. CSC is supported in part by the STFC Consolidated Grant ST/J000426/1 and by the grant 101-2112-M-007-021-MY3 of the National Science Council, Taiwan.

\section{References}

[1] C. -S. Chu and S. -L. Ko, "Non-abelian Action for Multiple Five-Branes with Self-Dual Tensors," JHEP 1205 (2012) 028 [arXiv:1203.4224 [hep-th]].

[2] E. Witten, "Some comments on string dynamics," In *Los Angeles 1995, Future perspectives in string theory* 501-523 [hep-th/9507121].

[3] J. M. Maldacena, "The Large N limit of superconformal field theories and supergravity," Adv. Theor. Math. Phys. 2 (1998) 231 [hep-th/9711200].

[4] P. S. Howe and E. Sezgin, "D = 11, p = 5," Phys. Lett. B 394 (1997) 62 [hep-th/9611008].

P. S. Howe, E. Sezgin and P. C. West, "Covariant field equations of the M theory five-brane," Phys. Lett. B 399 (1997) 49 [hep-th/9702008].

[5] M. Perry, J. H. Schwarz, "Interacting chiral gauge fields in six-dimensions and Born-Infeld theory," Nucl. Phys. B489 (1997) 47-64. [hep-th/9611065].

[6] M. Aganagic, J. Park, C. Popescu, J. H. Schwarz, "World volume action of the M theory five-brane," Nucl. Phys. B496 (1997) 191-214. [hep-th/9701166].

[7] P. Pasti, D. P. Sorokin, M. Tonin, "On Lorentz invariant actions for chiral p forms," Phys. Rev. D55 (1997) 6292-6298. [hep-th/9611100].

P. Pasti, D. P. Sorokin, M. Tonin, "Covariant action for a D = 11 five-brane with the chiral field," Phys. Lett. B398 (1997) 41-46. [hep-th/9701037].

I. A. Bandos, K. Lechner, A. Nurmagambetov, P. Pasti, D. P. Sorokin, M. Tonin, "Covariant action for the superfive-brane of M theory," Phys. Rev. Lett. 78 (1997) 4332-4334. [hep-th/9701149].

[8] C. S. Chu and D. J. Smith, "Multiple Self-Dual Strings on M5-Branes," JHEP 1001 (2010) 001 [arXiv:0909.2333 [hep-th]].

[9] C. -S. Chu, "A Theory of Non-Abelian Tensor Gauge Field with Non-Abelian Gauge Symmetry G x G," arXiv:1108.5131 [hep-th].

[10] P. -M. Ho, K. -W. Huang, Y. Matsuo, "A Non-Abelian Self-Dual Gauge Theory in 5+1 Dimensions," JHEP 1107 (2011) 021. [arXiv:1104.4040 [hep-th]].

[11] H. Samtleben, E. Sezgin and R. Wimmer, "(1,0) superconformal models in six dimensions," JHEP 1112 (2011) 062 [arXiv:1108.4060 [hep-th]]. 
[12] H. Samtleben, E. Sezgin, R. Wimmer and L. Wulff, "New superconformal models in six dimensions: Gauge group and representation structure," PoS CORFU 2011 (2011) 071 [arXiv:1204.0542 [hep-th]].

H. Samtleben, E. Sezgin and R. Wimmer, "Six-dimensional superconformal couplings of non-abelian tensor and hypermultiplets," JHEP 1303 (2013) 068 [arXiv:1212.5199 [hep-th]].

[13] I. Bandos, H. Samtleben and D. Sorokin, "Duality-symmetric actions for non-Abelian tensor fields," arXiv:1305.1304 [hep-th].

[14] J. Bagger and N. Lambert, "Modeling multiple M2's," Phys. Rev. D 75 (2007) 045020 [arXiv:hep-th/0611108].

A. Gustavsson, "Algebraic structures on parallel M2-branes," arXiv:0709.1260 [hep-th].

J. Bagger and N. Lambert, "Gauge Symmetry and Supersymmetry of Multiple M2-Branes," Phys. Rev. D 77 (2008) 065008 [arXiv:0711.0955 [hep-th]].

[15] O. Aharony, O. Bergman, D. L. Jafferis and J. Maldacena, " $\mathrm{N}=6$ superconformal Chern-Simons-matter theories, M2-branes and their gravity duals," JHEP 0810 (2008) 091 [arXiv:0806.1218 [hep-th]].

[16] C. -S. Chu, S. -L. Ko, and P. Vanichchapongjaroen, "Non-Abelian Self-Dual String Solutions," JHEP 1209 (2012) 018 [arXiv:1207.1095 [hep-th]].

[17] C. -S. Chu and P. Vanichchapongjaroen, "Non-abelian Self-Dual String and M2-M5 Branes Intersection in Supergravity," arXiv:1304.4322 [hep-th].

[18] V. Niarchos and K. Siampos, "M2-M5 blackfold funnels," JHEP 1206 (2012) 175 [arXiv:1205.1535 [hep-th]].

[19] C. M. Hull, "Exact pp wave slutions of eleven-dimensional supergravity," Phys. Lett. B 139 (1984) 39.

[20] A. A. Tseytlin, "Harmonic superpositions of M-branes," Nucl. Phys. B 475 (1996) 149 [hep-th/9604035].

[21] M. R. Douglas, "Branes within branes," In *Cargese 1997, Strings, branes and dualities* 267-275 [hep-th/9512077].

[22] E. Witten, "Small instantons in string theory," Nucl. Phys. B 460 (1996) 541 [hep-th/9511030].

[23] O. Barwald, N. D. Lambert and P. C. West, "On the energy momentum tensor of the M theory five-brane," Phys. Lett. B 459 (1999) 125 [hep-th/9904097].

[24] N. Lambert and P. Richmond, "(2,0) Supersymmetry and the Light-Cone Description of M5-branes," JHEP 1202 (2012) 013 [arXiv:1109.6454 [hep-th]].

[25] A. Fayyazuddin and D. J. Smith, "Localized intersections of M5-branes and four-dimensional superconformal field theories," JHEP 9904 (1999) 030 [hep-th/9902210]. 
D. J. Smith, "Intersecting brane solutions in string and M-theory," Class. Quant. Grav. 20 (2003) R233 [arXiv:hep-th/0210157].

[26] C. -S. Chu and D. J. Smith, "Towards the Quantum Geometry of the M5-brane in a Constant C-Field from Multiple Membranes," JHEP 0904 (2009) 097 [arXiv:0901.1847 [hep-th]].

[27] C. S. Chu and P. M. Ho, "Noncommutative open string and D-brane," Nucl. Phys. B 550 (1999) 151 [arXiv:hep-th/9812219].

C. S. Chu and P. M. Ho, "Constrained quantization of open string in background B field and noncommutative D-brane," Nucl. Phys. B 568 (2000) 447 [arXiv:hep-th/9906192]. C. S. Chu, "Noncommutative open string: Neutral and charged," arXiv:hep-th/0001144.

[28] V. Schomerus, "D-branes and deformation quantization," JHEP 9906 (1999) 030 [hep-th/9903205].

[29] N. Seiberg and E. Witten, "String theory and noncommutative geometry," JHEP 9909 (1999) 032 [arXiv:hep-th/9908142].

[30] A. Basu and J. A. Harvey, "The M2-M5 brane system and a generalized Nahm's equation," Nucl. Phys. B 713 (2005) 136 [arXiv:hep-th/0412310].

[31] C. -S. Chu and G. S. Sehmbi, "D1-Strings in Large RR 3-Form Flux, Quantum Nambu Geometry and M5-Branes in C-Field," J. Phys. A 45 (2012) 055401 [arXiv:1110.2687 [hep-th]].

[32] M. R. Douglas, "On D=5 super Yang-Mills theory and $(2,0)$ theory," JHEP 1102 (2011) 011. [arXiv:1012.2880 [hep-th]].

[33] N. Lambert, C. Papageorgakis, M. Schmidt-Sommerfeld, "M5-Branes, D4-Branes and Quantum 5D super-Yang-Mills," JHEP 1101 (2011) 083. [arXiv:1012.2882 [hep-th]].

[34] O. Aharony, M. Berkooz, S. Kachru, N. Seiberg, and E. Silverstein, "Matrix description of interacting theories in six-dimensions," Adv.Theor.Math.Phys. 1 (1998) 148157, [arXiv:hep-th/9707079 [hep-th]].

[35] O. Aharony, M. Berkooz, and N. Seiberg, "Light-cone description of $(2,0)$ superconformal theories in six dimensions," Adv. Theor. Math. Phys. 2 (1998) 119153, [arXiv:hep-th/9712117 [hep-th]].

[36] N. Arkani-Hamed, A. G. Cohen, D. B. Kaplan, A. Karch, and L. Motl, "Deconstructing (2,0) and little string theories," JHEP 01 (2003) 083, [arXiv:hep-th/0110146 [hep-th]].

[37] R. S. Ward, "Integrable and solvable systems, and relations among them," Phil. Trans. Roy. Soc. Lond. A 315 (1985) 451. 
[38] C. Saemann and M. Wolf, "On Twistors and Conformal Field Theories from Six Dimensions," J. Math. Phys. 54 (2013) 013507 [arXiv:1111.2539 [hep-th]].

L. J. Mason, R. A. Reid-Edwards and A. Taghavi-Chabert, "Conformal Field Theories in Six-Dimensional Twistor Space," J. Geom. Phys. 62 (2012) 2353 [arXiv:1111.2585 [hep-th]].

C. Saemann and M. Wolf, "Non-Abelian Tensor Multiplet Equations from Twistor Space," arXiv:1205.3108 [hep-th].

C. Saemann, "M-Brane Models and Loop Spaces," Mod. Phys. Lett. A 27 (2012) 1230019 [arXiv:1206.0432 [hep-th]].

C. Saemann and M. Wolf, "Six-Dimensional Superconformal Field Theories from Principal 3-Bundles over Twistor Space," arXiv:1305.4870 [hep-th]. 